\title{
Current knowledge on severe acquired brain injury with disorders of consciousness
}

\author{
Olivia Gosseries ${ }^{1,2,3}$ \& Steven Laureys ${ }^{1}$ \\ ${ }^{1}$ Coma Science Group, Cyclotron Research Centre and Neurology Department, University and University Hospital of Liege, Liege, Belgium, ${ }^{2}$ Center \\ for Sleep and Consciousness, Department of Psychiatry, University of Wisconsin, Madison, WI, USA, and ${ }^{3}$ Postle Laboratory, Department of \\ Psychology and Psychiatry, University of Wisconsin, Madison, WI, USA
}

This special issue of Brain Injury is designed to meet the needs of both scientific and clinical experts, as well as new learners in the field of severe brain injury and disorders of consciousness. The goal of this special issue is thus to present a number of studies focusing on patients in vegetative state/ unresponsive wakefulness syndrome and minimally conscious state, who are referred to as patients with disorders of consciousness. Most presented articles deal with diagnostic issues and functional neuroimaging advanced technology. A correct diagnosis of the level of consciousness is of paramount importance as it has profound impact on prognosis, treatment and care management. Diagnosis based only on clinical consensus has, however, been shown to induce a high rate of misdiagnosis [1]. Complementary neuroimaging techniques are therefore useful to increase the chance of giving an accurate diagnosis at the individual level [2].

The first article of this special issue, by Gosseries et al [3], gives an overview on recent advances in disorders of consciousness with a special focus on the diagnosis. Two cases of patients diagnosed at the bedside as being in a vegetative state/unresponsive wakefulness syndrome are reported along with their neuroimaging results that show contradictory results (i.e. one patient is in fact conscious). Once we know a patient is (minimally) conscious, one could ask what does it mean exactly? In the second article, Kotchoubey et al. [4] address this fundamental question as it is still not clear if patients in a minimally conscious state have temporal fluctuations in awareness or if they present a different kind of consciousness, or if it is a combination of both.

The assessment of the level of consciousness can be complicated with secondary medical problems, which diminish the chance to detect signs of consciousness at the patient's bedside. For instance, epilepsy is very common in patients with severe brain injury and sometimes it can be undetectable at the clinical level. In the third article of this special issue, Boly and Maganti [5] discuss how to monitor epilepsy in the intensive care unit. They also provide the current state of facts and potential interest of high-density EEG in detecting epilepsy.

Detection of consciousness also depends on the materials that are used at the bedside. To evaluate visual pursuit, which is one of the first signs of consciousness to recover after a coma, Thonnard et al. [6] report in this issue that it is best to employ a mirror in both the horizontal and the vertical axes. In the fifth study of this issue, Habbal et al. [7] show that, using electromyography, it is possible to detect voluntary responses via micromovements that are impossible to observe clinically at the patient's bedside. These two confirmatory studies on a large sample of patients $[6,7]$ are necessary to determine the reproducibility of previously published results $[8,9]$ in order to validate these tools before implementation in a clinical routine.

A major effort has also been devoted to setup ancillary markers to evaluate the brain's ability to generate consciousness without requiring the patients' collaboration. In this context, combining different neuroimaging techniques can be useful to assess the level of consciousness in a single patient. Napolitani et al. [10] review in this special issue studies that employed transcranial magnetic stimulation combined with high-density EEG in various altered states of consciousness. They show that this technique is reliable to evaluate and to quantify the level of consciousness at the individual level. The potential use of transcranial magnetic stimulation coupled with functional magnetic resonance imaging to assess consciousness in patients with disorders of consciousness is also reviewed in the seventh article written by Guller and Giacino [11].

One important question remains, however, regarding the use of different neuroimaging techniques for the diagnosis of the level of consciousness in patients with disorders of consciousness. How can we truly estimate the clinical utility of these techniques in the case of covert awareness? In other words, how can we trust the results of neuroimaging data in the absence of a true (behavioural) standard? Cruise et al. [12] propose in this issue some guidelines to follow to estimate the clinical utility of neuroimaging techniques.

Finally, the two last articles of this special issue are dedicated to the topic of pain that is closely related to the diagnosis (i.e., unconscious patients should not perceive pain whereas conscious patients do). Chatelle et al. [13] discuss pain issues in disorders of consciousness. Demertzi et al. [14] report the attitudes towards pain and end-of-life issues in 
patients with locked-in syndrome, patients who are easily misdiagnosed as being in an altered state of consciousness.

In conclusion, the 10 articles contained within this issue are reflective of the ongoing research on severe acquired brain injury with disorders of consciousness. We hope that this special issue provides the readers with an opportunity to advance their knowledge and skills of this challenging field.

\section{Acknowledgements}

This was funded by the Belgian National Funds for Scientific Research (FNRS), Fonds Léon Fredericq, James S. McDonnell Foundation; the Belgian American Educational Foundation (BAEF), Wallonie-Bruxelles International (WBI) Mind Science Foundation; European Commission; Concerted Research Action; Public Utility Foundation "Université Européenne du Travail" and "Fondazione Europea di Ricerca Biomedica'. OG received support from NIH grant MH064498 and MH095984 to Bradley R. Postle and from Giulio Tononi. OG is postdoctoral researcher and SL is research director at FNRS.

\section{Declaration of interest}

The authors report no conflicts of interest. The authors alone are responsible for the content and writing of this article.

\section{References}

1. Stender J, Gosseries O, Bruno M, Charland-Verville V, Vanhaudenhuyse A, Demerzti A, Chatelle C, Thibaut A, Heine L, Soddu A, et al. Diagnostic precision of multimodal neuroimaging methods in disorders of consciousness - a clinical validation study. The Lancet 2014; Published online http://dx.doi.org/10.1016/ S0140-6736(14)60042-8.
2. Laureys S, Schiff ND. Coma and consciousness: Paradigms (re)framed by neuroimaging. Neuroimage 2012;61(2):478-491.

-3. Gosseries O, Zasler N, Laureys O. Recent advances in disorders of consciousness: Focus on the diagnosis. Brain Inj 2014;28(9): $1141-1150$.

4. Kotchoubey B, Vogel D, Lang S, Muller F. What kind of consciousness is minimal? Brain Inj 2014;28(9):1156-1163.

-5. Boly M, Maganti R. Monitoring epilepsy in the intensive care unit: Current state of facts, and potential interest of high density EEG. Brain Inj 2014;28(9):1151-1155.

6. Thonnard M, Wannez S, Keen S, Brédart S, Bruno M-A, Gosseries O, Demertzi A, Thibaut A, Chatelle C, Charland-Verville V, et al. Detection of visual pursuit in patients in minimally conscious state: A matter of stimuli and visual plane? Brain Inj 2014;28(9): 1164-1170.

-7. Habbal D, Gosseries O, Noirhomme Q, Renaux J, Lesenfants D, Bekinschtein T, Majerus S, Laureys S, Schnakers C. Volitional electromyographic responses in disorders of consciousness. Brain Inj 2014;28(9):1171-1179.

8. Vanhaudenhuyse A, Schnakers C, Bredart S, Laureys S. Assessment of visual pursuit in post-comatose states: Use a mirror. J Neurol Neurosurg Psychiatry 2008;79(2):223.

9. Bekinschtein TA, Coleman MR, Niklison J, Pickard JD, Manes FF. Can electromyography objectively detect voluntary movement in disorders of consciousness? Journal of neurology, neurosurgery, and psychiatry 2008;79(7):826-828

10. Napolitani M, Bodart O, Canali P, Seregni F, Laureys S, Rosanova M, Massimini M, Gosseries O. Transcranial magnetic stimulation combined with high-density EEG in altered states of consciousness. Brain Inj 2014;28(9):1180-1189.

11. Guller Y, Giacino J. Concurrent TMS-fMRI: Potential applications to traumatic brain injury and disorders of consciousness. Brain Inj 2014;28(9):1190-1196.

12. Cruise D, Gantner I, Soddu A, Owen A. Lies, damned lies, and diagnoses: Estimating the clinical utility of assessments of covert awareness in the vegetative state. Brain Inj 2014;28(9): 1197-1201.

13. Chatelle C, Thibaut A, Whyte J, De Val M, Laureys S, Schnakers C. Pain issue in disorders of consciousness. Brain Inj 2014;28(9): 1202-1208.

14. Demertzi A, Jox R, Racine E, Laureys S. A European survey on attitudes towards pain and end-of-life issues in the locked-in syndrome. Brain Inj 2014;28(9):1209-1215. 